\title{
LA CONSTRUCCIÓN COMPARATIVA EN LA LENGUA DE GÓNGORA
}

\author{
Mario Garcia-Page
}

UNED

1. La gramática española adolece aún de un estudio exhaustivo y pormenorizado que dé cuenta de la diversidad de construcciones comparativas que existen en español ${ }^{1}$. Tal ausencia puede deberse, en gran medida, a la decisión de algunas

1 Quizás los estudios más completos sobre la comparativa en español son los muy recientes trabajos de S. GUTIERREZ (Estructuras comparativas, Madrid, Arco/Libros, 1994; y Estructuras pseudocomparativas, Madrid, Arco/Libros, 1994) y la última versión, ampliada y modificada, de «Construcciones y sintagmas comparativos en el español actual», de J.A. MARTfNEZ, publicada en Cuestiones marginadas de gramática española, Madrid, Istmo, 1994, 115-72 (orig. en In Memoriam Inmaculada Corrales, Univ. de La Laguna, 1987, 1, 319-36 y, con el título «Oraciones consecutivas y comparativasm, en Lecciones del / y /I Curso de Lingülstica Funcional (1983-1984), Univ. de Oviedo, 1985 , 141-51). Vid., además, D.L. BOLNGER, «The comparison of inequality in Spanish», Lg, 26, 1950, 28-62; O. Prytz, *Construcciones comparativas en espafiol», RRo, 14, 1979, 260-78; Á. LOPEZ GARClA, «La comparación en español: estructura fraseológica y estructura oracional», Serta Philologica. F. Lázaro Carreter, Madrid, Castalia, 1983, 1, 315-27; T. ESPAÑol, «Estructuras de la oración comparativa en español», AF, 11-13, 1986, 109-118; E. ACIN, «Construcciones con como en español modemo», RILCE, 3:1, 1987, 25-57; J. Miyoshi, «Cinco fómulas de expresión del MAS comparativo», Lingüfstica Hispánica, 12, 1990, 57-70; - «Estudios sobre las expresiones de los comparativos en el español modernow, Acta Humanistica et Scientifica Songyo Kyotiensis, 19:3, 1990, 198 279; - «Construcción MAS QUE DE EL ( , LO) QUE», Lingülstica Hispánica, 14, 1991, 83-92; - «Término español MAS: comparativo y conjunción adversativa», The Bulletin of the Intemational Institute for Linguistic Sciences, 11, 1992, 125-42; - «El TD de la construcción comparativa», Lingǘstica Hispánica, 15, 1992 (en prensa); A.M. VAzQUEZ, «Algunas 
gramáticas básicas del español y ciertos estudiosos de tratarlas como una subclase de oraciones consecutivas ${ }^{2}$, e, incluso, de asociarlas, por lo que puedan tener de relación, con otros tipos de configuraciones sintácticas: relativas, modales, coordinadas, partitivas ${ }^{3}, \ldots$ En las precarias y escuetas observaciones que dedican a las mismas ciertas gramáticas se mezclan criterios distintos y se llega a hacer distinciones poco fértiles, como la separación que propone la Academia ${ }^{4}$ entre comparativas de modo y comparativas de cantidad; propuesta criticada por otros gramáticos, como Gili Gaya ${ }^{5}$, quien, a cambio, sugiere la división binaria entre comparativas de cualidad y comparativas de cantidad; división que tampoco tiene plena utilidad por cuanto que desconoce estructuras que no comparan cualidades o cantidades. Sánchez Márquez ${ }^{6}$ propone la división tripartita: modalidad, cantidad y cualidad. Algunos lingüistas ${ }^{7}$ se limitan a señalar las distintas categorías o funciones (sustantivo, adjetivo, sujeto,...) que pueden compararse; aspecto interesante que, de estudiarse concienzudamente, podría arrojar alguna luz sobre ciertos tipos de construcciones aún poco explorados y sus comportamientos sintácticos, así como las posibilidades de distribución de los términos comparados (o de los propios morfemas discontinuos de la comparación).

observaciones sobre la denominación y clasificación de las oraciones comparativas», Congreso de la Sociedad Española de Lingülstica. XX Aniversario, Madrid, Gredos, 1990, 1, 797-804; E. MoNTOLfo, «La comparación de igualdad como procedimiento semiótico: La estructura 'ser+como+SN'n, Investigaciones Semióticas IV, Madrid, Visor, II, 1992,933-942; S. GUTIÉRREZ, «MÁS DE - MÁS QUE», Contextos, X: 19-20, 47-86; -Las odiosas comparaciones, Logroho, Consejería de Cultura, Deportes y Juventud, 1992. A esta lista hay que añadir la bibliografía citada en otras partes del trabajo, especialmente en n. 14. Cfr., además, R. RivarA, «How many comparative are there?", Linguistics, 163, 1975, 35-41.

2 P.e., RAE, Gramática de la lengua española, Madrid, Espasa Calpe, 1931, 389; R. SECo, Manual de gramática española, Madrid, Aguilar, 1992, 243-5; F. RIVERA, «Sobre el parentesco histórico y estructural de las comparativas de igualdad y las consecutivas de intensidad», Alfinge, 3, 1985, 115-24; J.A. MARTf́neZ, op.cit., 161-72; A.I. Álvarez MENÉndeZ, «Consecutivas y construcciones comparativas», Las construcciones consecutivas en español, Oviedo, Univ. Oviedo, 1989 , cap. 5.

3 Vid., entre otros, E. AlARCOS LLORACH, «Español /que/»(1963), Estudios de gramática funcional del español, Madrid, Gredos, ${ }^{3} 1980,260-74$, esp. 272-4; Á. LOPEZ GARCIA, op. cit., 325 ss.; A. NARBONA, «Sobre las oraciones bipolares», Alfinge, 1, 121-39, esp. 126; J. MARTINEZ ÁlvAREZ, «Conectores complejos en español», Lecciones del I y II Curso de Gramática Funcional, Oviedo, Univ. Oviedo, 131-40, esp. 135-6; E. Franchini, Las condiciones gramaticales de la coordinación copulativa en español, Francke Verlag Bem, Románica Helvética 102, 209-37; J. A. MARTtNEZ, o. c., esp. 137-61; S. GUTIÉRRE, Estructuras pseudocomparativas, passim...

4 Esbozo de una nueva gramática de la lengua española, Madrid, Espasa Calpe, 1979, 543-6.

5 Curso superior de sintaxis española (11*ed.), Barcelona, Bibliograf, 1976, 316-8.

6 M.J. SÁNCHEZ MÁRQUEZ, Gramática moderna del español. Teoría y norma, Buenos Aires, Ediar, 1982, 244-6.

7 P.e., A. Bello, Gramática de la lengua castellana, Madrid, Arco/Libros, 1988, § 1.007; J. Alcina-J.M. BleCuA, Gramática española (3a ed.), Barcelona, Ariel, 1982, 1042-3. 
2. Para el propósito de este trabajo, que es fundamentalmente dar cuenta de las distintas posiciones distribucionales que consiguen los términos de la comparación y los morfemas discontinuos (tan...como, más/menos...que, etc.) en la lengua de Góngora -muestra representativa de las preferencias de la época, siglos XVI y XVII - partimos de la distinción de varias formas de construcciones comparativas:

1) El adjetivo es el núcleo de la comparación entre dos SSNN: «ioh bella Galatea $\left[\mathrm{SN}_{1}\right]$, más süave [SA] / que los claveles que tronchó la Aurora $\left[\mathrm{SN}_{2}\right]$ ! $(629)^{8}$.

2) Dos adjetivos aplicados a un mismo sustantivo son los términos de comparación. La estructura resultante es un «sintagma comparativo»: «veloz, intrépida ala $/$-menos cansado $\left[\mathrm{A}_{1}\right]$ que confuso $\left[\mathrm{A}_{2}\right]$ - escala» (635).

En casos como éste (víd. también grupo 3), Fernandez Ramírez ${ }^{9}$ supone que los términos de la oposición (tú y santo, en «(tú) tienes más razón que un santo», coinciden con términos de comparación: «Tienen más de criminales que de penitentes», «hallamos más bien pintura que escultura», etc.).

3) Los términos de comparación son dos complementos «geminados» (en la terminología de Alcina-Blecua ${ }^{10}$ ): están representados por la misma categoría sintagmatica (SP, $\mathrm{SN}, \ldots$ ) y función $(\mathrm{CC}, \mathrm{CD}, \ldots)$ : «feroz ardiente muestra / hicieron dos robustos luchadores / de sus músculos, menos defendidos / de el blanco lino $\left[\mathrm{SP}_{1}\right]$ que del velo obscuro $\left[\mathrm{SP}_{2}\right] »(659)^{11}$.

8 La cifra entre paréntesis que aparece en los ejemplos del corpus corresponde a la página de la obra consultada: L. DE GÓNGORA, Obras completas, Madrid, Aguilar, 1972.

9 Gramática española. 3.1. El nombre, Madrid, Arco/Libros, \& 81, esp. 79 y n. 176.

10 Op. cit., 1043 y 1059.

11 Bien es verdad que esta triple división elemental podría incluso simplificarse a dos grupos, el 2 y 3: en 3 se incluirfa el 1 como una clase particular. Repárese en que, siendo el adjetivo el núcleo de comparación, éste relaciona dos SSNN geminados que suelen funcionar de sujeto. No obstante, considerando los tres grandes grupos diferenciados - si bien el tercero un tanto heterogéneo-, la comparación es posible entre agrupaciones sintácticas más complejas (p.e., oraciones). A. Alonso y P. HenRIQUez UREÑa (Gramática castellana. $l$ (11' ed.), Buenos Aires, Losada, $1973,54-5)$ sefialan un tipo de comparación entre dos grupos configurados por sustantivo y adjetivo, a cuyo esquema podrian responder textos como «No de tu media luna / ha sido, Amor, flechada / saeta más alada / que la ausencia importuna» (601) o «Dos son las chozas, pobre su artificio / más aún que caduca su materia» (688). Como sugieren algunos gramáticos, en algunas de las llamadas estructuras comparativas «oracionales» en las que uno de los grupos comparados presenta tácitamente callado el elemento que es común al otro, parece gramaticalmente posible la recuperación de éste: «de tal suerte, que quedaban, / más que en los anzuelos peces / entre sus cabellos almas» (49), «privilegios, el mar a quien di redes / más que a la selva lazos Ganimedes» (678), «el Júpiter novel, de más coronas / ceñido que sus orbes dos de zonas» (696), «Sobre corchos después, más regalado / sueño le solicitan pieles blandas, / que al príncipe entre holandas, 
3. Asimismo, se tienen en cuenta las diversas formas de distribución que pueden presentar los términos de comparación, así como los morfemas que suelen encabezarlos ${ }^{12}$. Para la delimitación de las diversas pautas de construcción, seleccionamos el intensivo más (más...que) como modelo para la notación general. No en balde la comparativa de superioridad es la estructura más recurrente; v.gr.:

1) Construcción comparativa «normal» en la que los morfemas se disponen de modo discontinuo (más...que): [más A] [que B]. Cabe pensar que este tipo de construcción es el más frecuente en el español estándar, el que ha consolidado el uso a lo largo de la historia; v.gr.: «No pues de aquella sierra engendradora / más de fuerzas que de cortesía» (637): [más de fuerzas] [que de cortesía].

2) Construcción comparativa "continua», en la que los morfemas, por algún tipo de inversión o desplazamiento, aparecen en la secuencia de forma continua, i.e., inmediatamente consecutivos (...más que...). Aunque es una construcción en extremo recurrente en la lengua artística de la Edad de Oro, no parece que domine sobre la construcción «normal» («discontinua»), al menos en Góngora $^{13}$. Su empleo es también conocido en el lenguaje de todos los días, de modo especial en el coloquio o en determinados contextos linguísticos o pragmáticos (p.e., estructuras estereotipadas del tipo «come más que una lima»14; elativizaciones como «Este chico no es tonto, es más que tonto» ${ }^{15}$; etc.).

/púrpura tiria o milanés brocadon (638), «Endeche el siempre anado esposo muerto / con voz doliente tan sorda queja tiene la soledad como el desierto» (507), «De el Sandoval, que a Denia aun más corona / de majestad que al mar de muros ella" (690); v.gr.: quedaban más almas entre sus cabellos que peces [quedaban] en los anzuelos, [yo] di al mar más redes que Ganimedes [dio] lazos a la selva, etc. Pero no siempre existe un término común que pueda eludirse por sobreentenderse en uno de los grupos: «que al mar debe con término prescrito / más sabandijas de cristal que a Egipto / horrores deja el Nilo que le baña» (685) [se comparan las cantidades número de «sabandijas»número de «horroresm], «Más [besos], Clori, que he tejido / jazmines al cabello desatado, / y más besos te pido / que abejas tuvo el escuadrón armadow (580), etc. Otras estructuras complejas, como kque pacen campos, que penetran senos / de las ondas no menos / aquellos perdonados / que de la tierra estos admitidos» (688), cabría describir dentro del grupo tercero, si los subrayados se analizan como SSNN (sujeto) geminados.

12 Vid. J.A. MAYORAL, «Sobre construcciones comparativas en el lenguaje poético de los siglos XVI y XVIl*, Estudios Filologicos. En Homenaje a Eugenio de Bustos Tovar, Salamanca, Univ. Salamanca, 1992, 2, 641-56 (y, en este volumen, una versión reducida y ligeramente distinta). El citado autor limita su estudio a las comparativas de superioridad estereotipadas configuradas por dos SSNN con núcleo adjetivo.

13 Como ya sugeriamos en M. GaRCfa-PAGE, «Notas a La lengua poética de Góngora, de Dámaso Alonson, $A E F, 17,1994,201-222$; cfr. J.A. Mayoral, o.c., 652.

14 Para el español, véanse, entre otros, W. Beinhauer, El espafiol coloquial, Madrid, Gredos, ${ }^{3} 1985,241$-3 y 297-325; J. SKULTETY, «El reino animal en las comparaciones fraseol6gicas», Philologica (Bratislava), 29, 1981, 91-107; - «Algunas observaciones sobre las comparaciones 
La comparativa continua presenta dos variedades:

2.1) [A más] [que B]: se extrapola el primer término de la comparación anteponiéndose al intensivo más: «sangre, más que una morcilla, / honra, más que un paladín» $(137)=$ [sangre, más] [que una morcilla] - [honra, más] [que un paladín].

2.2) [más [que B] A]: el primer sintagma de la comparativa queda escindido por la intrusión del segundo inmediatamente a continuación del intensivo: «porque su bruñida frente / y sus mejillas se hallan / más que roquete de obispo / encogidas y arrugadas» $(60)=$ [más [que roquete de obispo] encogidas y arrugadas].

Cabe reparar en la semejanza entre los procedimientos de consecución de este tipo de comparativas (continuas) y los tradicionales hipérbatos por inversión del orden o por estrangulamiento sintáctico de una estructura sintagmática, como puede verse en los siguientes textos (en los que la separación del determinante respecto de su núcleo nominal se debe a la interposición del verbo y un sintagma comparativo): «dulce tranquilidad que este (mora / tan grato como pobre] albergue, donde / sellado el labio, la quietud se esconde» (592) o sólo del grupo comparativo: «Deste [más que la nieve] blanco toro» (457). De ahí que las comparativas continuas (en especial, las del grupo 2.2) puedan describirse como manifestaciones particulares del hipérbaton; un tipo de hipérbaton omitido precisamente por uno de los estudiosos más conocedores de los recursos formales de Góngora: Dámaso Alonso ${ }^{16}$.

Cabe igualmente indicar que en latín ya se conocía la construcción comparativa que presenta agrupados los morfemas de comparación (magis/minus/

españolas desde el punto de vista fraseológico y fraseográfico», Beiträge zur Romanischen Philologie, 25:2, 1986, 163-77; I. TAMBA-MECZ, «Comparaisons hyperboliques», Le sens figuré, Paris, PUF, 1981, 1447; A. ZULUAGA, «Locuciones elativas», Introducción al estudio de las expresiones fijas, Frankfurt, Verlag Peter D. Lang, 1980, 146-9; J. CH. DE VAN PRAGG, «Intensidad expresiva de las comparaciones estereotipadas» (abstract), IV Congreso Internacional de Hispanistas, Salamanca, Univ. de Salamanca, 1982, 1, 815-6; J.M. GONZALEZ CALvo, «Sobre la expresión de «lo superlativo» en español (II)», AEF, 8, 1985, 114-46, esp. 137 ss.; M. GARClA-PAGE, «Frases elativas», Congreso de la Sociedad..., cit., 1, 485-96; - «Más sobre la comparativa fraseologica en español» (en prensa); G. ORTEGA, «Comparaciones estereotipadas y superlatividad», Congreso de la Sociedad..., cit., 2, 729-37; J. MIYOSHI, «Expresiones superlativas con los comparativos», Hispánica, 34, 1990, 142-54; H. AYALA, «La hipérbole popular: los más y los menos», Paremia, 2, 1994, 239-44.

15 V., p.e., M. GARClA-PAGE, «Frases elativas», cit., 486-7.

16 Entre los estudios de D. ALONSo sobre el hipérbaton, pueden verse La lengua poética de Góngora (3* ed.), Madrid, CSIC, 1961. Anejo XX de RFE, 177 212; - Vida y obra de Medrano, 
plus...quam): «plus quam se atque suos amauit omnes» (Catulo, LVIII, vs. 3) ${ }^{17}$, «proicere aptavit potius quam talia Cretam» (Catulo, LXIV, vs. 82), o el marcador conjuntivo del segundo término, junto al verbo malo o al adverbio malle: «mavult quam Syrias Britanniasque» (Catulo, XLV, vs. 22), «Nulli se dicit mulier me nubere malle / quam mihi, non si se Iuppiter ipse petat» (Catulo, LXX, vss. 1-2); «Lesbius est pulcer: quid ui? quem Lesbia malit / quam te cum tota gente, Catulle, tua» (Catulo, LXXIX, vss. 1-2).

Si en la poesía latina se destacaba como un fenómeno recurrente, es presumible que el grado de intensidad alcanzado en los Siglos de Oro se deba fundamentalmente a la influencia renacentista y especialmente al modelo petrarquista, tal como sucede con otras convenciones artísticas. A esta razón habría que sumar otras posibles causas, como el tipo de género y las convenciones métricas (ritmo, tipo de estrofa y de verso,...), etc.

3) Construcción «inversa" por permutación del orden de aparición de los términos comparados: [que B] [más A]. Este tipo de construcción hiperbática - sin duda, menos frecuente que las anteriores en el lenguaje de los siglos XVI y XVII- no se produce, al parecer según nuestro análisis, en la obra poética de Góngora, pero sí en la de otros escritores de la misma época: «prudentísima Ester, que el sol más bella» (Cervantes, $997^{18}$ ) [que el sol] [más bella]; «Virgen, que el sol más pura» (fray Luis de León, $263^{19}$ ): [que el sol] [más pura].

4. El carácter totalmente arbitrario de la manera en que el poeta construye las comparativas queda manifiesto en textos como 1-2), en los que aparecen ensartados dos tipos distintos de comparativas según el esquema distribucional:

(1) ¡Oh bella Galatea, MÁS süave QUE los claveles que tronchó la Aurora; [más A] [que B] blanca MÁS QUE las plumas de aquel ave [A más] [que B] que dulce muere y en las aguas mora (629)

Madrid, CSIC, 1948, cap. 16; - Poesía, de F. DE MEDRANo, Madrid, Cátedra, 1988, 93-112. Cfr. nuestras «Notas a La lengua poética de Góngora, de DÁmASO AlONSO», o.c.

17 Catulli Carmina, Barcelona, Aurelia Romero ed.

18 Obras completas, Madrid, Aguilar, 1970; cit. en M. GARCIA-PAGE, «El cultismo sintáctico en Cervantes» (en prensa) y en J. A. Mayoral, o.c., 654. Véanse además: M. GarCia-PaGe, *Algunas notas sobre la «lengua poética» de Cervantes», Actas I Cong. Int. AEC (Almagro, 1991; en prensa); «Usos y valores del adjetivo en Cervantes», Actas IV Coloquio Int. AEC (Alcalá de Henares, 1991; en prensa); «Estructuras de «sintaxis inversa» en Cervantes», Estudios Filológicos. En Homenaje a Eugenio de Bustos Tovar, cit.,1, 327-47, esp. 340-1; «Tipología del hipérbaton en Cervantes», Recherches en Linguistique Hispanique (Actas Ve Colloque de Linguistique Hispanique, Aix-en-Provence, 1992), Univ. de Provence, Études Linguistiques 22, 1994, 269-79, esp. $276-7$.

19 Obras completas castellanas, Madrid, BAC, 1967, vol. 2; cit. en J.A. MAYORAL, o.c., 654. 
(2) Sacro pastor de pueblos, que en florida

edad, pastor, gobiernas tu ganado,

$M A ́ S$ con el silbo QUE con el callado

y MÁS QUE con el silbo con la vida (481) [más [que B] A]

[más A] [que B]

5. Clase de morfemas discontinuos (intensivos). El valor comparativo de antes.

La mayoría de las gramáticas señala, según indiquen igualdad, superioridad o inferioridad, los siguientes morfemas de comparación: tan(to)...como, igual / más / menos...que, etc. Algunos lingüistas añaden certeramente mejor y peor, como ya ocurría en latín: «Las colores muertas /resucita el son; /toman el latón / mejor que el acero» (429), «que a dos Sarmientos, cada cual glorioso,/ obedeció mejor que al bastón grave» (474), «Mejor se la darán que en las ajenas en la casa de Luna» (547). Otros, más bien pocos, apuntan la posibilidad de que otro tipo de «partículas» (normalmente, adverbios) puedan funcionar como marcadores de comparación. Esta facultad parece poseerla el adverbio antes $^{20}$, correlativo de que, cuando, despojado de su valor temporal, equivale semánticamente al intensificador comparativo más, con el valor de «prioridad», "preferencia». En los contextos en que aparece antes, éste puede ser sustituido por el signo más. Así, antes comparte los mismos esquemas distribucionales que más; v.gr.: [antes A][que B]: «lágrima antes enjuta que llorada» (667), «tiernos rayos en una piedra dura / de un sol antes caduco que luciente» (449); [A antes][que B]: «invidioso aun antes que vencido, / carbunclo ya en los cielos engastado» (559); [antes [que B] A]: «siempre sonante a aquel, cuya memoria / antes peinó que canas desengaños» (494).

Así mismo, como el más, antes puede recibir la modificación intensificadora de otros adverbios como aún, incluso, todavia; comp.: «Ilustran obeliscos las ciudades, / a los rayos de Júpiter expuesta / -AUN MÁS que a los de Febosu corona» (659), «iOh mar!, quien otra vez las ha fiado / de tu fortuna AUN MÁs que de su hado» (666) - «Camisa al Centauro fue su vida / AUN ANTES abrasada que vestida»(591), «invidioso AUN ANTES que vencido...»(599).

$\mathrm{El}$ adverbio antes presenta además otras características; p.e., no puede sustituirse por menos sin que se altere el significado (incluso, posiblemente la

20 Véase especialmente M. GARCfA-PAGE, «Antes comparativo» (en prensa). Este valor de antes ya lo indicó R.J. Cuervo en su Diccionario de Construcción y Régimen de la Lengua Castellana, París, A.Roger y F. Chemoviz, 1886, 1, 173, n. 10, s.v. antes; y a él se han referido, entre otros, J.A. MARTINEZ, op.cit., 151-2; S. GARCIA, «Sobre algunos adverbios temporales + que», Revista de Filología (Univ. La Laguna), 10, 1991, 159-74, esp. 165-9; y S. GUTIÉRREZ, Estructuras pseudocomparativas, 58-60. Cfr; también el estudio de TH. DIETHERICH-D.J. NAPOLI ( «Comparative rathem, $J L, 18,1982,137-65$ ), aunque no todas las observaciones pueden aplicarse al antes español. 
estructura sintáctica). Generalmente liga dos sintagmas homogéneos dentro de una estructura no oracional (o sin verbo) ${ }^{21}$.

6. Las pautas distribucionales señaladas ( $\$ 3$ ) son, aparentemente, modificadas al interpolarse, entre los términos configuradores de la estructura comparativa, elementos extraños a la misma: «abrirán paso a cuantos peregrinos I tan libres podrán ya como devotos / besando el mármol desatar sus votos* $(607)=$ [más A] + $\mathrm{x}+$ [que B]; «menos quizás dio astillas / que ejemplos de color a estas orillas» (673), «menos tiene de musa que de arpia» (542), «Agradecida Amarilis, / flores las abejas más / deberán a su coturno / que al novillo celestial» (234) $=[$ más $+x+A][$ que $B]$.

Esta presunta alteración se muestra más intensa que los casos de las comparativas «continuas», donde los morfemas que debieran aparecer inmediatamente seguidos son distanciados, separados por un signo ajeno a los términos de comparación. Generalmente, la intercalación del término intruso se efectúa en el primer grupo comparativo, bien, respecto del esquema 2.1), entre el intensificador más y su término: $[\mathrm{A}+\mathrm{x}+$ más] [que $\mathrm{B}]=$ «carbunclo ya en los cielos engastado / en bordadura pretendió tan bella / poco rubi ser más que mucha estrella» (599), «ni amor que de singular / tenga más que de infiel» (271); bien entre los dos grupos de la comparación: [A más] + $\mathbf{x}+$ [que $\mathrm{B}$ ] = «en vuestra banda más preso / que en las redes del amon (391), «la bella infanta, a quien le debe albores / tantos, la Aurora, como rosicleres (611); bien en distintas posiciones: $[\mathrm{A}+\mathrm{x}+$ más] $+\mathrm{x}+$ [que $\mathrm{B}]=$ «mientras que a cada labio, por cogello,/ siguen más ojos que al clavel tempranon (447). Lo mismo cabría decir de las modificaciones del segundo esquema, 2.2), como, p.e.: «que ausencia haga un garzón / más que yo, sí, venturoso / pero más amante no» (236), «antes peinó que canas desengaños» $(494)=[$ más [que $+x+B] A],[$ más $+x$ + [que B] A],...

Sin embargo, la ruptura de la continuidad es sólo aparente, ya que, no obstante la incorporación del nuevo elemento, no varía el orden distribucional de los grupos conformantes de la comparación.

7. Agrupando los dos aspectos arriba indicados, la clase léxica o sintáctica de los elementos que conforman la comparación y el esquema distribucional que adoptan, pueden aducirse los ejemplos siguientes según los distintos esquemas:

1) [más A][que B] (construcción «normal»)

21 Véase M. GARCfA-PAGE, «Antes comparativo*, cit.; cfr. S. GUTtÉRRE, Estructuras pseudocomparativas, cit., 58-60. 
1.1) Construcción en la que un adjetivo funciona de núcleo de la comparación entre dos SSNN:

(3) ¡Oh bella Galatea, más süave

que los claveles que tronchó la Aurora (629)

1.2) Construcción en la que se comparan dos adjetivos aplicados a un mismo nombre:

(4) suelo menos barrido que regado (540)

1.3) Dos grupos sintagmáticos geminados se ponen en relación de comparación. Son diversas las funciones sintácticas que pueden desempeñar tales grupos (CD, CA, etc.):

(5) blanco coro de Náyades lascivas precie más vuestras sombras fugitivas que una verde margen de escondida fuente (454)

(6) velas de Occidente, que, más de joyas que de viento llenas (573)

(7) menos de aljaba que de red armado (674)

2) Construcción comparativa «continua»

2.1) [A más][que B]

2.1.1) Construcción en la que un adjetivo funciona como núcleo de la comparación entre dos SSNN:

(8) [Galatea] blanca más que las plumas de aquel ave que dulce muere $y$ en las aguas mora (629)

(9) Grandes, más que elefantes y que abadas (459)

(10) Gallardo, más que la palma que besa el aire sereno salio Fileno (388)

(11) Dulce, más que el arroyuelo que las azucenas pisa llegó Belisa (387)

(12) donde, aún cansado más que el caminante concurría el camino (649)

2.1.2) Construcción en la que se comparan dos cualidades de un mismo objeto: 
(13) Quejas de un pescadorcillo, honor de aquella ribera, que una roca solicita, sorda tanto como bella (197)

(14) estrellas fragantes, más que claras la noche ve (227)

(15) invidioso aun antes que vencido carbunclo ya en los cielos engastado (599)

2.1.3) Construcción en la que se comparan dos sintagmas categorial y funcionalmente equivalentes ( $\mathrm{CD}, \mathrm{CC}, \mathrm{CA}, \mathrm{Ady}, \ldots)$ :

(16) tu claustro verde, en valle profanado de fiera menos que de peregrino (507)

(17) quien me fuerza a que huya de su prision, dejando mis cadenas rastro en tus ondas más que en tus arenas (666)

(18) antiguo descubrieron blanco muro por sus piedras no menos que por su edad majestuosa cano (530)

(19) Gastando, pues, en tanto la memoria ajena envidia más que propia cera, por el Carmen la lleva a la Victoria (544)

(20) Humos reconocí en Su Chimenencia de abstinente no menos que de avaro ( 551 )

2.2) [más [que B] A]

2.2.1) Construcción en la que un adjetivo funciona como núcleo de los términos (SSNN) que se comparan:

(22) ciudad más que ninguna populosa (572)

(23) Deste más que la nieve blanco toro (457)

(24) Parca criel, más que las tres severa (591)

2.2.2) Construcción en la que se ponen en relación de comparación dos adjetivos aplicados a un mismo nombre. No he encontrado ningún ejemplo en el corpus.

2.2.3) Construcción en la que se comparan dos grupos sintagmáticos geminados complementos de un mismo núcleo: 
(25) que el soberano Tapia

hizo (más que en árboles, en bronces, piedras, mármoles),

en su verso eternice su prosapia (566)

(26) y [gobiernas...] más que con el silbo con la vida (481) 